\title{
Écrivons ensemble les nouvelles pages de la revue !
}

\section{Let's Write the New Pages of the Review together!}

\section{S. Perrot (Président de la SFETD)}

(C) Lavoisier SAS 2018

Avec ce premier numéro de 2018, nous sommes heureux de pouvoir ouvrir de nouvelles pages de la revue pour y écrire de nouveaux chapitres. Cet éditorial est l'occasion de déclarer, avec plaisir et enthousiasme, Douleur et Analgésie, revue officielle de la Société française d'étude et de traitement de la douleur (SFETD).

Une page qui s'ouvre pour la SFETD, pour répondre à un développement indispensable à une société savante :

- une société scientifique doit pouvoir disposer d'un organe officiel pour présenter les travaux de ses membres, dans leur diversité et foisonnement ;

- une société savante doit aussi attirer vers elle d'autres scientifiques, de tous horizons, nationaux et internationaux, pour des échanges et dialogues novateurs et enrichissants.

De nouvelles pages pour Douleur et Analgésie, pour un nouvel élan :
- de nouveaux membres au comité éditorial ;

- de nouvelles sections dans la revue pour accompagner le foisonnement de la recherche en douleur;

- de nouveaux projets de développement, en français et en anglais, pour valoriser les actions scientifiques de la SFETD.

Au nom du conseil d'administration de la SFETD, je tiens à remercier François Cesselin, rédacteur en chef, d'avoir accepté sans hésiter les propositions de la SFETD et cette nouvelle orientation de Douleur et Analgésie, journal déjà reconnu dans le monde francophone. Un journal, comme une société savante, doit savoir évoluer, s'adapter sans cesse à de nouveaux défis. Nous appelons les abonnés et lecteurs fidèles de la revue, ainsi que les membres de la SFETD, à lire, mais aussi écrire ensemble les nouvelles pages de $\mathrm{Dou}$ leur et Analgésie, cette belle revue qui est maintenant la nôtre et à tous.

\footnotetext{
S. Perrot $(\bowtie)$

Centre de la douleur, hôpital Cochin,

27, rue du Faubourg-Saint-Jacques,

F-75014 Paris, France

e-mail : serge.perrot@aphp.fr
} 